\title{
AGAVE ALBOPILOSA (AGAVACEAE, SUBGÉNERO LITTAEA, GRUPO STRIATAE), UNA ESPECIE NUEVA DE LA SIERRA MADRE ORIENTAL EN EL NORESTE DE MÉXICO
}

\author{
Ismael Cabral Cordero¹, José Ángel Villarreal Quintanilla², Eduardo A. \\ Estrada CASTILlón ${ }^{1}$ \\ ${ }^{1}$ Universidad Autónoma de Nuevo León, Facultad de Ciencias Forestales \\ Apdo. postal 41, 67700 Linares, Nuevo León, México. \\ icabralc@gmail.com - icabralc@prodigy.net.mx \\ 2Universidad Autónoma Agraria Antonio Narro, Departamento de Botánica \\ 25315 Buenavista, Saltillo, Coahuila, México.
}

\section{RESUMEN}

Se propone como especie nueva a Agave albopilosa, un maguey pequeño rupícola del grupo Striatae, de la Sierra Madre Oriental. Su característica más sobresaliente es un mechón circular de pelos blancos en la porción distal de las hojas, justo debajo de la espina terminal. Las flores son ligeramente campanuladas, similares a las de A. stricta, pero con lóbulos más cortos y también los frutos son más pequeños. Se incluye una ilustración de la planta y una clave para la separación de las especies del grupo.

Palabras clave: Agavaceae, Agave albopilosa, México, Sierra Madre Oriental.

\begin{abstract}
Agave albopilosa is described as a new species. It is a small plant, among the agaves of the Striatae group, growing in the Sierra Madre Oriental. Its most notorious feature is a ring of hairs near the end of the leaves, just below the terminal thorn. The flowers are characteristic of the group, more similar to the ones in A. stricta, slightly campanulate but with shorter lobes, and also a shorter fruit. An illustration and a key to separate the species of the group are provided.
\end{abstract}

Key words: Agavaceae, Agave albopilosa, Mexico, Sierra Madre Oriental. 


\section{INTRODUCCIÓN}

El grupo Striatae del género Agave fue propuesto originalmente por Baker en 1888 (Gentry, 1982). Sus características distintivas son las hojas estriadas, lineares y aserradas en el margen, las flores con tubos largos, lóbulos pequeños y el ovario sin prolongarse en un cuello, proyectado hacia el interior del tubo. Las especies (taxa) que conforman este conjunto en el tratado de agaves de Norteamérica de Gentry (1982) son: Agave striata Zucc. ssp. striata, A. striata Zucc. ssp. falcata (Engelm.) Gentry, A. dasylirioides Jacobi \& Bouché y A. stricta Salm-Dyck, todas ellas dadas a conocer en el siglo antepasado.

Recientemente se han descrito de la misma afinidad a A. tenuifolia Zamudio \& E. Sánchez (1995), A. petrophila García-Mend. \& E. Martínez (1998a y b) y A. rzedowskiana P. Carrillo et al. (2003). La novedad aquí propuesta adiciona una especie a este grupo.

Agave albopilosa I. Cabral, Villarreal \& A. E. Estrada, sp nov. (Figs. 1-3)

Agave strictae similis sed differt foliis $14-23 \mathrm{~cm}$ longis 8-14 mm latis, parum incurvatis, apicibus caespite trichomatum albidorum ante spinam terminalem; floribus breviter campanulatis, tubis 8-10 mm longis lobis 4-5 mm longis erectis parum incurvatis; capsulis oblongis 10-12 mm longis 8-10 mm latis.

Planta perenne, cespitosa; tallos decumbentes; raíz leñosa, de hasta $20 \mathrm{~cm}$ de largo y de 2 a $2.5 \mathrm{~cm}$ de diámetro en la parte inicial, que se ancla en la roca; roseta simétrica, hemisférica, de 20 a $40 \mathrm{~cm}$ de diámetro y 15 a $36 \mathrm{~cm}$ de alto, hojas 100 a 180 por planta, arregladas en 15 a 25 hileras, linear-triangulares, de 14 a $23 \mathrm{~cm}$ de largo y 8 a 14 mm de ancho en la parte media, adelgazándose en el ápice, base ensanchada, envainante, ápice terminado en un mechón anular, oblicuo, con pelos blancos de 4 a $7 \mathrm{~mm}$ de largo, localizado 4 a $6 \mathrm{~mm}$ debajo de la espina, espina recta a sigmoidea, negruzca, de 3-6 mm de largo y 0.7 a $0.9 \mathrm{~mm}$ de ancho en la base, subcónica, segmento entre la espina y el mechón de pelos con margen entero y cubierto por pelos aplicados, margen aserrado, rígido, ligeramente curvadas, ligeramente cóncavas en forma de "v" abierta, con costilla dorsal, estriadas en ambas superficies, glabras, verde amarillentas a ligeramente glaucas; inflorescencia en escapo floral espigado, pedúnculo de 30 a $50 \mathrm{~cm}$ de largo y 6 a $9 \mathrm{~mm}$ de diámetro, recto a inclinado, con brácteas esparcidas linear-triangulares, de 3 a $5 \mathrm{~cm}$ de largo y 3 a $5 \mathrm{~mm}$ de ancho, ápice acuminado, de color amarillento, espiga cilíndrica, de 15 a $30 \mathrm{~cm}$ de largo y 


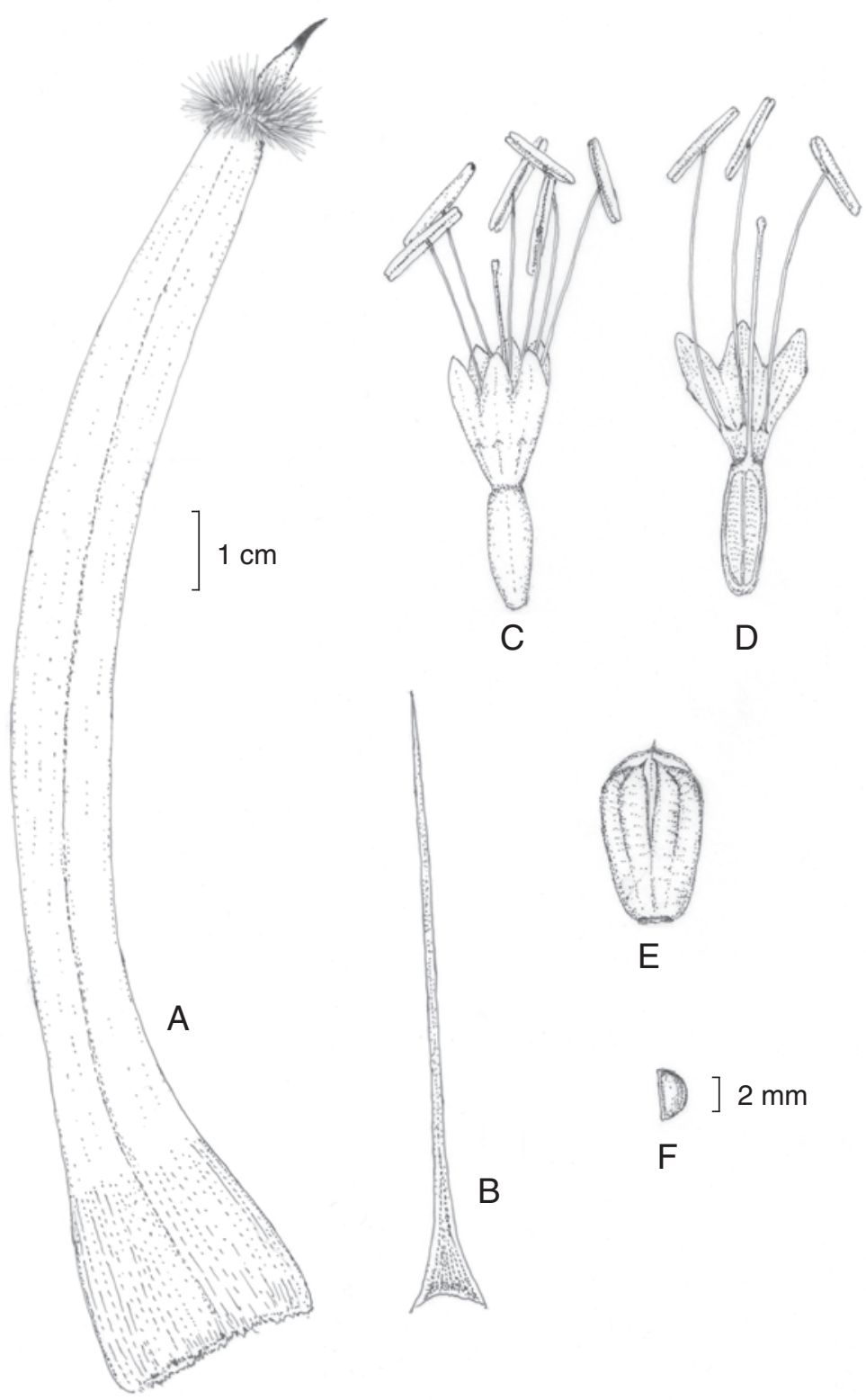

Fig. 1. Agave albopilosa I. Cabral, Villarreal \& A. E. Estrada. A. hoja; B. bráctea; C. flor; D. disección de la flor que muestra la parte interna; E. fruto; F. semilla. La escala de $1 \mathrm{~cm}$ es válida para las figuras A-E. 


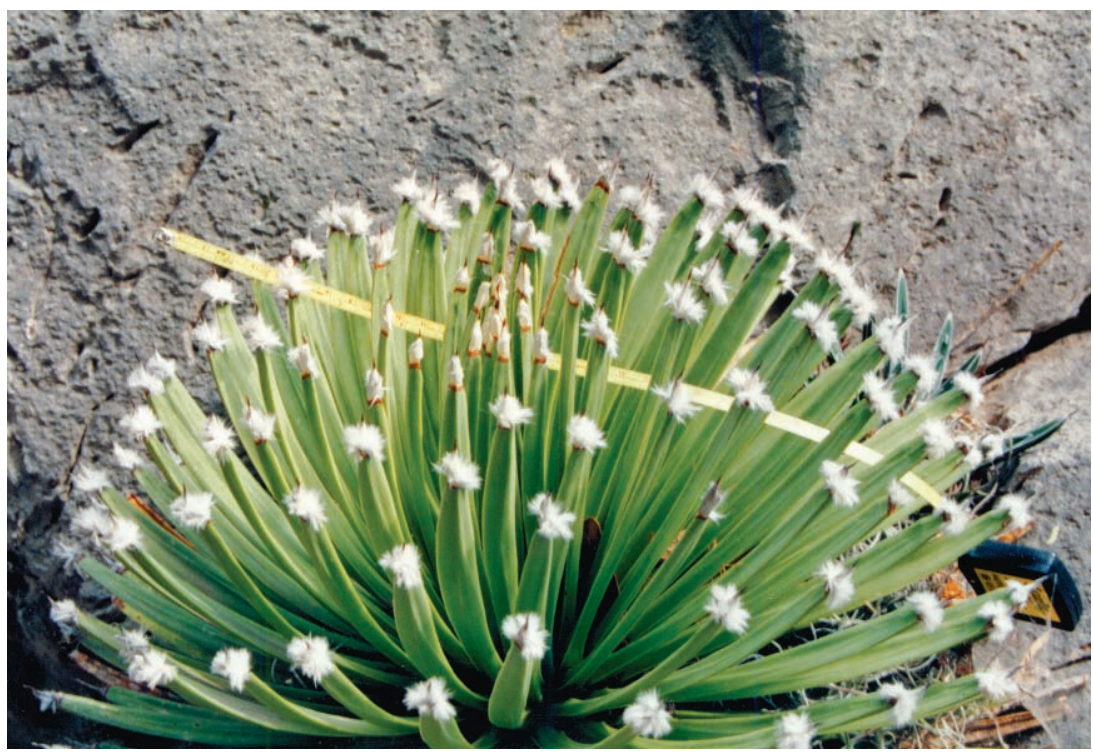

Fig. 2. Agave albopilosa I. Cabral, Villarreal \& A. E. Estrada. Planta con hojas mostrando el mechón de pelos.

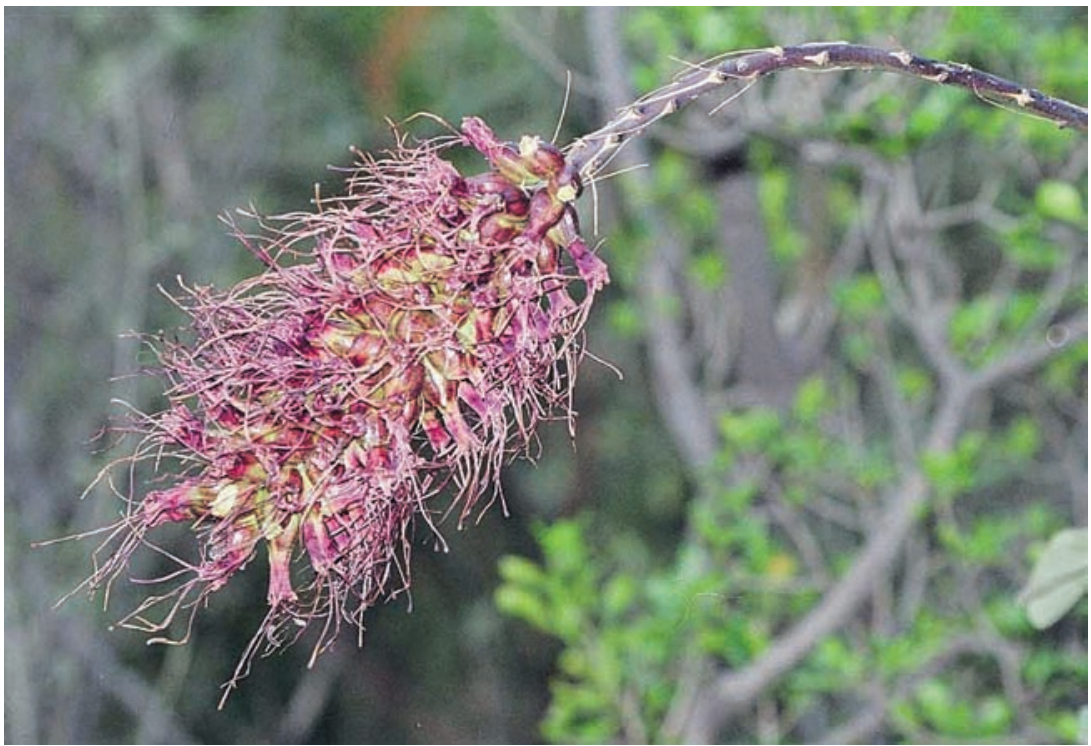

Fig. 3. Inflorescencia de Agave albopilosa I. Cabral, Villarreal \& A. E. Estrada. 
4 a $8 \mathrm{~cm}$ de diámetro, densa; botones florales verde purpúreos, flores 70 a 120 por inflorescencia, usualmente pareadas, ligeramente campanuladas, verdosas, con tintes purpúreos, brácteas iguales a las del escapo, pero más cortas; ovario cortamente oblongo, de 8 a $10 \mathrm{~mm}$ de largo, verde, perianto de 14 a $16 \mathrm{~mm}$ de largo, tubo de 8 a $10 \mathrm{~mm}$ de largo, lóbulos de 4 a $5 \mathrm{~mm}$ de largo, erectos, ligeramente incurvados, estambres insertos en la parte media del tubo, exertos, filamentos aplanados, de 25 a $30 \mathrm{~mm}$ de largo, verde amarillentos, anteras de 8 a $10 \mathrm{~mm}$ de largo, amarillas; estilo cilíndrico, de 26 a $32 \mathrm{~mm}$ de largo, verdoso, estigma ligeramente trilobulado; cápsulas oblongas, de 10 a $12 \mathrm{~mm}$ de largo, de 8 a $10 \mathrm{~mm}$ de ancho, con estrías transversales, glabras, cafés oscuras; semillas semicirculares, aplanadas, engrosadas en el lado curvo, de 3 a 3.5 mm de largo y 1.5 a 2 mm de ancho, lisas, de color negro.

Tipo: México. Sierra Madre Oriental, 8.VII.2004, I. Cabral 1612 (Holotipo: MEXU, isotipos: ANSM, CFNL, ENCB, UNL).

Material adicional consultado: Sierra Madre Oriental. 24.VI.1998. J. Verduzco 324 (ANSM).

La especie es conocida de un área restringida de la Sierra Madre Oriental, crece sobre taludes rocosos en poblaciones dispersas formadas por individuos solitarios o pequeñas colonias en altitudes de 1000 a $1500 \mathrm{~m}$. Comparte el habitat con especies como Agave bracteosa S. Watson, A. lechuguilla Torr., A. striata Zucc., A. victoriaereginae Moore, Hechtia texensis S. Watson, Dasylirion berlandieri S. Watson, Acacia berlandieri Benth., Mortonia greggii A. Gray, Abelia coriacea Hemsl., Purshia plicata (D. Don) Henr. y Fraxinus greggii A. Gray, entre otras. Dada su distribución restringida y escasa abundancia, es una especie amenazada que deberá protegerse y propagarse en jardines botánicos y viveros para asegurar su existencia. Para evitar la colecta ilegal, la localidad precisa se mantiene oculta.

Taxonómicamente la especie se ubica en la sección Striatae y en su morfología se relaciona con A. stricta. El hábito y las hojas son semejantes, aunque éstas son menos numerosas y llevan un mechón de pelos blancos en la porción terminal. Las flores son más pequeñas (20-25 mm vs. 24-35 mm) con los lóbulos del perianto más cortos (4-5 mm vs. 8-10 mm), y cápsulas más cortas (10-12 mm vs. 12-14 mm). Difieren también en el hábitat (rupícola vs. terrestre) y la distribución (noreste de México vs. Puebla). Otra especie con la que presenta similitud morfológica es $A$. rzedowskiana, en la que los lóbulos del perianto son más grandes (7-8 mm vs. 4-5 mm en A. albopilosa) y falta la pubescencia en las hojas. 
El epíteto específico describe el círculo de pelos blancos que rodean la espina terminal de las hojas, característica distintiva del nuevo taxon. Localmente conocida como mechudo.

Clave para la separación de las especies del grupo Striatae

1. Flores cilíndricas; lóbulos del perianto de $1 / 3$ a 1/4 de largo del tubo.

2. Inflorescencia laxa; filamentos de los estambres de 20 a $25 \mathrm{~mm}$ de largo, cortamente exertos; hojas aprox. 50, de hasta $130 \mathrm{~cm}$ de largo A. tenuifolia

2. Inflorescencia densa; filamentos de los estambres de 20 a $50 \mathrm{~mm}$ de largo; largamente exertos; hojas aprox. 100, de 25 a $60 \mathrm{~cm}$ de largo A. striata

1. Flores campanuladas; lóbulos del perianto de la mitad del largo del tubo.

3. Hojas de 40 a $100 \mathrm{~cm}$ de largo; rosetas laxas; flores amarillo-verdosas.

4. Flores de 20 a $25 \mathrm{~cm}$ de largo; filamentos insertos en el ápice del tubo floral; hojas 40 a $70 \mathrm{~cm}$ de largo A. petrophila

4. Flores de 25 a $40 \mathrm{~cm}$ de largo; filamentos insertos en la parte media del tubo floral; hojas de 40 a $100 \mathrm{~cm}$ de largo A. dasylirioides

3. Hojas de 20 a 35(-50) cm de largo; rosetas densas; flores rojas a rojizas.

5. Flores de 24 a 35 mm de largo; cápsulas de 12 a 14 mm largo; sur de Puebla A. stricta

5. Flores de 20 a $25 \mathrm{~mm}$ de largo; cápsulas de 10 a $12 \mathrm{~mm}$ de largo.

6. Hojas con un mechón de pelos en la parte distal; lóbulos del perianto de 4 a $5 \mathrm{~mm}$ de largo; Sierra Madre Oriental A. albopilosa

6. Hojas sin mechón de pelos; lóbulos del perianto de 7 a 8 mm de largo; Sierra Madre Occidental (Jal., Sin.) A. rzedowskiana

\section{AGRADECIMIENTOS}

Agradecemos la revisión del escrito a Abisaí García Mendoza, la descripción en latín a Guy Nesom. La ilustración se debe a la colaboración de Miguel A. Carranza Pérez. El grupo Taller de Tecnología Prehistórica del Noreste de México, en especial Héctor Lazcano Fernández y Raúl Femat Lozano, así como Mario Mansilla Terán, Martín Bremer Bremer, Mario Chapa y Ulises Treviño Treviño, integrantes de la Red Desarrollo ConCiencia, apoyaron al primer autor en las excursiones de campo. El Consejo Nacional de Ciencia y Tecnología otorgó una beca a Ismael Cabral para realizar los estudios de posgrado en la Facultad de Ciencias Forestales de la Universidad Autónoma de Nuevo León. 


\section{LITERATURA CITADA}

Carrillo, P., R. Vega y R. Ramírez. 2003. Agave rzedowskiana, a new species in subgenus Littaea (Agavaceae) from western Mexico. Brittonia 55(3): 240-244.

Gentry, H. S. 1982. Agaves of continental North America. University of Arizona Press. Tucson, Arizona. 670 pp.

García, A. y E. Martínez. 1998a. Una nueva especie de Agave, subgénero Littaea (Agavaceae) de Guerrero y Oaxaca, México. Sida 18(1): 227-230.

García, A. y E. Martínez. 1998b. Agave petrophila García-Mend. \& E. Martínez, nom. nov. (Agavaceae). Sida 18(2): 627.

Zamudio, S. y E. Sánchez. 1995. Una nueva especie de Agave del subgénero Littaea (Agavaceae) de la Sierra Madre Oriental, México. Acta Bot. Mex. 32: 47-52.

Recibido en octubre de 2006.

Aceptado en marzo de 2007. 